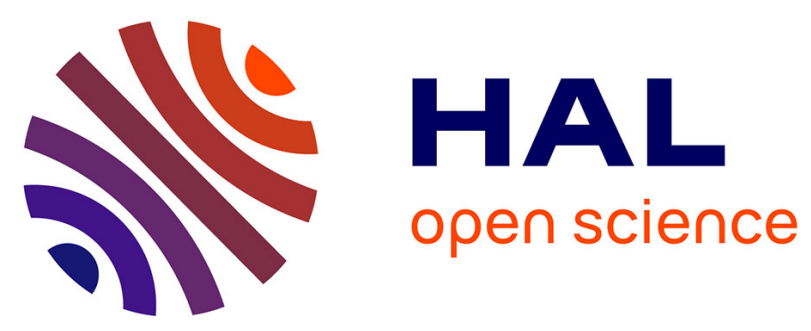

\title{
Directional Loudness of Low-Frequency Noises Actually Presented Over Loudspeakers And Virtually Presented Over Headphones
}

\author{
Gauthier Berthomieu, Vincent Koehl, Mathieu Paquier
}

\section{- To cite this version:}

Gauthier Berthomieu, Vincent Koehl, Mathieu Paquier. Directional Loudness of Low-Frequency Noises Actually Presented Over Loudspeakers And Virtually Presented Over Headphones. Journal of the Audio Engineering Society, 2019, 67 (9), pp.655-665. 10.17743/jaes.2019.0018 . hal-02294336

\section{HAL Id: hal-02294336 \\ https://hal.univ-brest.fr/hal-02294336}

Submitted on 25 Mar 2021

HAL is a multi-disciplinary open access archive for the deposit and dissemination of scientific research documents, whether they are published or not. The documents may come from teaching and research institutions in France or abroad, or from public or private research centers.
L'archive ouverte pluridisciplinaire HAL, est destinée au dépôt et à la diffusion de documents scientifiques de niveau recherche, publiés ou non, émanant des établissements d'enseignement et de recherche français ou étrangers, des laboratoires publics ou privés. 


\title{
Directional Loudness of Low-Frequency Noises Actually Presented Over Loudspeakers And Virtually Presented Over Headphones*
}

\author{
Gauthier Berthomieu, AND Vincent Koehl, AND Mathieu Paquier
}

Univ Brest, Lab-STICC, CNRS, UMR 6285, F-29200 Brest, France

\begin{abstract}
Previous studies have revealed that the loudness of low-frequency pure tones increased with the interaural time difference (ITD). This effect accounts for the directional loudness phenomenon at low frequencies, where ITD is the main localization cue. So far, this effect has only been studied for sounds generated and presented directly over headphones, which are not natural listening conditions. The present study aims at investigating this effect on lowfrequency noises stemming from real sources. Twenty subjects assessed the loudness of stimuli that were actually displayed by loudspeakers arranged at various locations within a listening room or that were recorded with a dummy head and virtually reproduced through headphones. Results show that the directional loudness sensitivity (DLS) is in agreement with the previously revealed ITD effect. Moreover, the DLS was higher when stimuli were reproduced over headphones than over loudspeakers, specifically when frontal sources were located at a short distance from the listeners. One hypothesis on this effect relies on visual cues that were available to the listeners only when sounds were reproduced over loudspeakers, providing information about the source distance. Listeners were also aware that sounds were reproduced on loudspeaker or headphones, possibly involving different loudness processings, leading to DLS differences.
\end{abstract}

\section{O INTRODUCTION}

\subsection{Effect of direction and ITD on loudness}

The direction of a sound source in relation to the listener affects significantly the loudness of the sounds it produces, especially in the horizontal plane [1, 2, 3, 4]. There is growing awareness that this phenomenon, called directional loudness, has to be taken into account for audio reproduction systems, especially for multichannel ones [5, 6]. This phenomenon is largely accounted for by at-ear pressures, that are highly dependent on the position of the source with respect to the listener. More precisely, the filtering applied by the head, torso and ears of the listener on a sound that reaches his/her ears depends on the direction from which the sound comes [5]. This filtering leads to a variation of the at-ear sound pressure level, which is the main factor involved in the loudness sensation. Consequently, directional loudness was mainly expected to occur for high-frequency stimuli, since the filtering effect of the body is rather small for low frequencies [7]. Nevertheless, directional loudness has been observed for low-frequency narrow-band noises

*Corresponding author: Tel: +331-98-01-65-86; e-mail: gauthier.berthomieu@univ-brest.fr
[2]. Furthermore, binaural loudness models that take at-ear pressures as input tend to underestimate directional loudness effect in comparison with experimental data [8, 2]. These observations suggested that at-ear sound pressure modifications could not fully explain the directional loudness phenomenon, especially for low-frequency stimuli.

This assumption was confirmed by previous studies that highlighted an effect of the interaural time difference (ITD) on the loudness of pure tones. A small but significant loudness increase $(+1.5 \mathrm{~dB})$ was reported for low-frequency (200 and $400 \mathrm{~Hz}$ ), low-level (40 phon) pure tones whose ITD reached $772 \mu$ s [9]. This ITD value corresponded to a $90^{\circ}$ incidence angle according to Kuhn's low-frequency model [10]. The loudness increase proved to be caused by the ITD itself rather than by the related localization. This was observed by estimating the loudness of sounds with a fixed ITD whose induced localization was counterbalanced thanks to an Interaural Level Difference (ILD, which is the second binaural localization cue) leading to the opposite ear [11]. Another previous experiment focused on the evolution of this effect as a function of the level of the stimulus [12]. ITD was reported to significantly enhance (between $1.5 \mathrm{~dB}$ and $2 \mathrm{~dB}$ ) the loudness of pure tones whose loudness level was up to 40 phon at $100 \mathrm{~Hz}$ and up to 50 phon 
at $200 \mathrm{~Hz}$. These results indicate that ITD is involved in the binaural loudness process. In order to control the ITD in these experiments, sounds were presented in an audiometric booth and reproduced over headphones with a fixed ITD applied between the two channels, which constitutes a rather unecological sound presentation.

\subsection{Effect of ecological validity on loudness}

According to Epstein and Florentine [13, 14], the ecological validity of an experiment is likely to affect the binaural loudness summation and should be taken into account in loudness research. This was concluded from results regarding the binaural-to-monaural loudness ratio which is the loudness growth observed when a sound is presented at the two ears compared to at one ear only. Such a comparison can be conveniently achieved by feeding either one channel or two channels of headphones. Assuming that a monaural listening situation was not ecologically valid to the listeners, Epstein and Florentine assessed the binauralto-monaural ratio for speech stimuli displayed whether on loudspeakers, whether on headphones [13], with or without visual cues [14]. They reported that the binaural-tomonaural loudness ratio decreased as the ecological validity of the presentation increased (that is for loudspeakerreproduced stimuli displayed with visual cues). Although binaural-to-monaural ratio is not directly related to directional loudness, they are both involved in binaural loudness summation and one could wonder if ecological validity could have an effect on directional loudness. Furthermore, Sivonen reported that reverberant sounds yielded to smaller directional loudness effects than anechoic ones [3]. Thus, the room effect is also likely to decrease the directional effects observed in an anechoic chamber.

These findings about binaural and directional loudness both suggest that effects observed under rather unecological conditions could be reduced in more realistic situations. Since the previous results about the effect of ITD on loudness were obtained with pure tones directly generated and displayed over headphones, one may wonder whether the loudness increase reported for headphone-generated stimuli lateralized with the sole ITD still holds under more realistic conditions, namely for real sources located in a room. Besides, stimuli directly synthesized over headphones are often perceived "within the head" rather than coming from a distant source [15], which could constitute an additional source of disparity between these laboratory presentation conditions and natural ones.

\subsection{Aim of the present study}

The present study has been designed in order to investigate the directional loudness effects for low-frequency noises stemming from loudspeakers located around the subject in a listening room. Contrarily to the previous results about the ITD effect, such stimuli were emitted by real sources in a room that was not anechoic. The stimuli were presented to the listeners by either actually reproducing them over loudspeakers or by virtually reproducing them over headphones, in order to study the influence of the presentation conditions. One should note that reproducing the stimuli either over loudspeakers or headphones is not likely to modify the directional effects as long as visual cues are available to the subjects. According to Sivonen et al., the directional loudness sensitivity of narrow-band noises was the same whether they were directly reproduced through visible loudspeakers located all around the subject in an anechoic room [2] or reproduced at the same positions with headphones by using individual HRTFs [16]. The latter experiment took place in the same anechoic chamber and the loudspeakers were still mounted in the tested directions even if sounds were reproduced over headphones to improve the plausibility of the binaural synthesis. In this specific case, it might not have been possible to distinguish between two identical sounds that were played back over loudspeakers or headphones [17]. However, in most laboratory experiments, binaural stimuli (whether recorded or synthesized) are not presented with visual representations of the sound sources. This also applies to the present study as no loudspeaker was mounted in the directions under study when sounds were reproduced over headphones. Thus, the presentation conditions under investigation differed not only because of the reproduction system itself (loudspeakers or headphones) but also because of the information they provided about the sound sources, such as visual information.

The effects of direction on loudness were assessed in terms of Directional Loudness Sensitivity (DLS) by comparing directional stimuli to frontal references. As the room effect is likely to alter the directional loudness [3] and as the position of a sound source is likely to evoke a specific room response, several spatial configurations of the sound sources in the listening room were tested so that the observed effects would not be caused by a specific loudspeaker layout within the room. The DLS was also assessed for headphone-reproduced stimuli lateralized with the sole ITD for comparison with previous studies.

\section{Material and methods}

The effect of direction on loudness (or DLS) was investigated by matching low-frequency noises emitted by lateral sources with frontal references. Two different presentation conditions were under study: one where sounds were actually played back over loudspeakers and one where these sounds were first binaurally recorded and then virtually reproduced through headphones. For the low-frequency noise under test, the room effect may vary with the source position and thus affect the directional loudness [3]. The DLS was then investigated for four different spatial configurations of the sound sources enabling to elicit and take into account different room responses.

\subsection{Stimulus}

The stimulus that was investigated in this experiment was a narrow-band noise, which bandwidth was set to one Equivalent Rectangular Bandwidth (ERB) so that all the spectral components of this noise were contained in one 
critical band. The center frequency of the noise was $265 \mathrm{~Hz}$ since previous experiments reported a predominant effect of ITD on loudness between 200 and $400 \mathrm{~Hz}$ [9, 12]. The ERB was computed according to the following equation by Glasberg and Moore [18]:

$$
\mathrm{ERB}_{N}=24.7 \times(4.37 F+1)
$$

where $F=0.265 \mathrm{kHz}$ is the center frequency of the ERB. This equation led to a $53-\mathrm{Hz}$ wide ERB. The narrow-band noise was generated according to the procedure described by Edmonds and Culling [19]. The reference level was fixed at 50 phon [12] (corresponding to $58.4 \mathrm{~dB} \mathrm{SPL}$ at $265 \mathrm{~Hz}$ according to the ISO 226 standard [20]) at the center of the head of the subject in his/her absence. The duration of each stimulus was $1.6 \mathrm{~s}$, smoothed by onset and offset ramps of $100 \mathrm{~ms}$.

\subsection{Procedure}

Twenty subjects aged from 20 to 25 years took part in the experiment and were payed for their participation. Their hearing thresholds were measured for the noise investigated in this experiment using a standard procedure [21] and had to be $\leq 10 \mathrm{~dB}$ HL to go on with the experiment. None of them had experience in laboratory listening tests.

Points of Subjective Equality (PSE, corresponding to the level at which a sound is perceived as loud as a reference sound) were obtained using a two-interval, twoalternative, forced choice paradigm (2I2AFC), following a 1-up 1-down rule [22]. In each trial, a test sound and a reference sound were presented in random order, separated by a $500 \mathrm{~ms}$ pause. The test sound was presented by a lateral source (azimuth of $-90^{\circ}$ or $+90^{\circ}$ ) and the reference sound was presented by a frontal source $\left(0^{\circ}\right)$. The task was to indicate whether the first or the second sound was louder, with no other possible option and independently of any other perceived difference. The software was developed and executed on MAXMSP and subjects responded using two buttons on a gamepad. These instructions were indicated orally and a few trials were presented before the beginning of the test in order to familiarize the subjects with the interface and procedure.

The test sounds were initially presented at a level randomly set $10 \mathrm{~dB}$ above or below the reference sound level. After the subject's response, their level was lowered by a given amount if the subject judged it was louder than the reference sound, whereas it was increased by a certain amount if the subject judged the reference sound was louder. The step size was initially set to $\pm 4 \mathrm{~dB}$ and changed to $\pm 1 \mathrm{~dB}$ after two reversals in the equalization curve (i.e. when the loudness judgment of the subject changed from one trial to another for the same test sound). A total of eight reversals were collected for each test sound. Then, PSEs were derived from the means of the test sound levels at the six last reversals, relative to the reference sound level. The experiment was split in two sessions, with a 5 min break between them. Each of these sessions consisted of the same task, but under different presentation conditions: one where sounds were actually played back over loudspeakers and one where sounds were virtually reproduced over headphones. Each subject participated in the two sessions in a random order.

\subsection{Actual presentation}

The experiment took place in a sound-attenuated listening room, which had been designed so that its RT60 was $0.3 \mathrm{~s}$ at $250 \mathrm{~Hz}$ [23], in compliance with the IEC 6026813 standard [24] concerning room characteristics for listening tests on loudspeakers. Four loudspeakers were placed at various locations within the room (see Fig. 1) to reproduce both reference and test sounds. As shown in Fig 2 , this setup enabled to arrange frontal and lateral loudspeakers in four different spatial configurations within the room to differently elicit its response. In one given configuration, the frontal $\left(0^{\circ}\right)$ loudspeaker playing reference sounds, denoted R, was located at either 1 or $2 \mathrm{~m}$ from the listening point whereas both distances could be allocated to the lateral loudspeakers $\left(-90^{\circ}\right.$ or $\left.+90^{\circ}\right)$ playing test sounds, denoted $\mathrm{T}$.

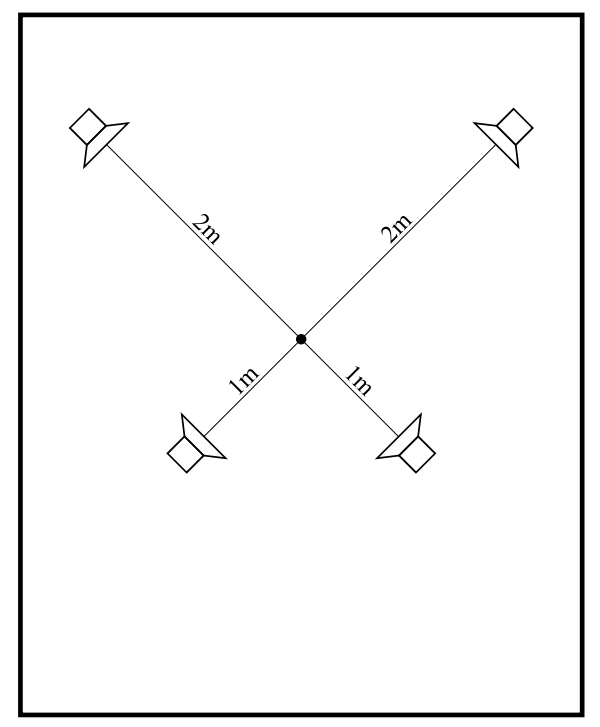

Fig. 1. Loudspeakers arranged around the listening point (dot) in the test room.

The subject sat in a chair that was placed in the room so that the center of his/her head corresponded to the listening point depicted by the dot in Fig. 1. The subject's head was precisely positioned using a headrest and a chin rest and it remained fixed during the procedure. Each spatial configuration was achieved by pointing the subject towards one of the four loudspeakers. The experiment was subsequently divided into four sub-sessions, with respect to the four spatial configurations under test. The sub-session order was randomly chosen for each subject. Each sub-session lasted between 5 and $10 \mathrm{~min}$ and subjects were allowed to stand up from the chair between each sub-session. The headrest and the chin rest were maintained in a fixed position during the whole session so that the subject's head could be precisely positioned in the same position at the beginning of each sub-session. 


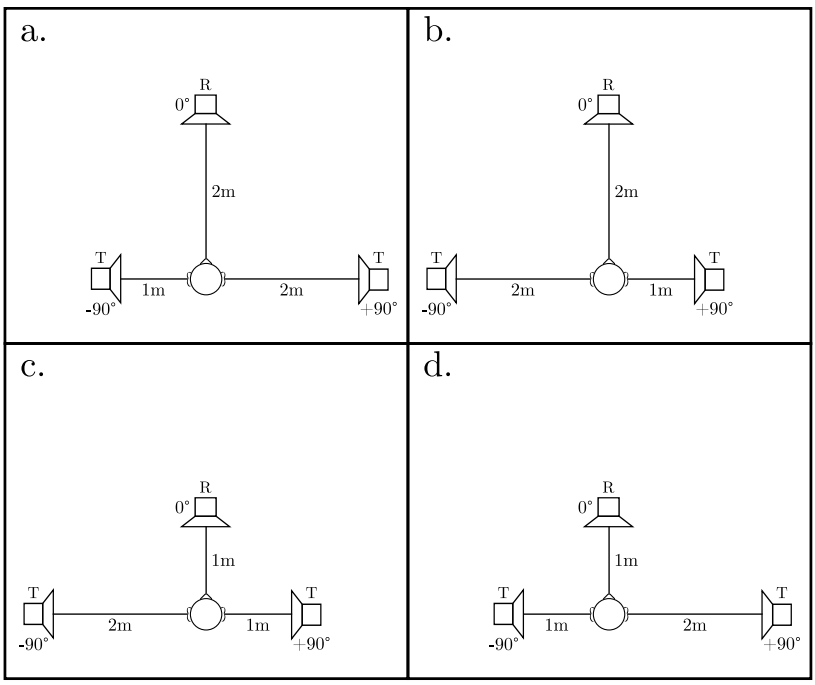

Fig. 2. Loudspeakers arrangement for each of the four spatial configurations. Sources denoted R display the reference sounds, sources denoted $\mathrm{T}$ display the test sounds.

Sounds were generated using a RME Fireface 800 sound card and reproduced through Amadeus PMX 4 loudspeakers, powered by Audac DPA154 amplifiers. The four loudspeakers were calibrated so that they produced a loudness level of 50 phon at the center of the head of the subject in his/her absence. In other words, they delivered $58.4 \mathrm{~dB}$ SPL [20] at the listening point whatever their location within the room.

\subsection{Virtual presentation}

The stimuli that were presented to the listeners through the actual sources were recorded in order to be played back through headphones in these presentation conditions. In order to provide the listener with the same acoustic stimuli with headphones as with loudspeakers, binaural recordings were carried out. At the frequency range under test, inter-individual variations of head size and shape cause small differences of at-ear pressures and arrival times [25] that are even smaller than the just noticeable differences [26]. Thus, the recordings were carried out with a dummy head (Neumann KU100) that enabled an accurate recording of the sounds at the entrance of the (blocked) ear canal. The dummy head was precisely arranged at the position of a subject's head during the actual presentation (see Fig. 3) and was calibrated by using a Brüel \& Kjær Type 4231 sound calibrator at $1 \mathrm{kHz}$. Thus, twelve stimuli were recorded this way corresponding to each of the three azimuths $\left(-90^{\circ}, 0^{\circ}\right.$ and $\left.90^{\circ}\right)$ in each of the four spatial configurations (Fig. 2).

The stimuli were presented over Sennheiser HD650 headphones (circumaural, open). The output level of the system was adjusted by placing the headphones on the calibrated dummy head. The session took place in the same listening room as in the loudspeaker session. Subjects sat in the same chair, but no loudspeaker was mounted in any of the tested directions so that subjects could not match the sounds they heard with an identifiable sound source. Sub-

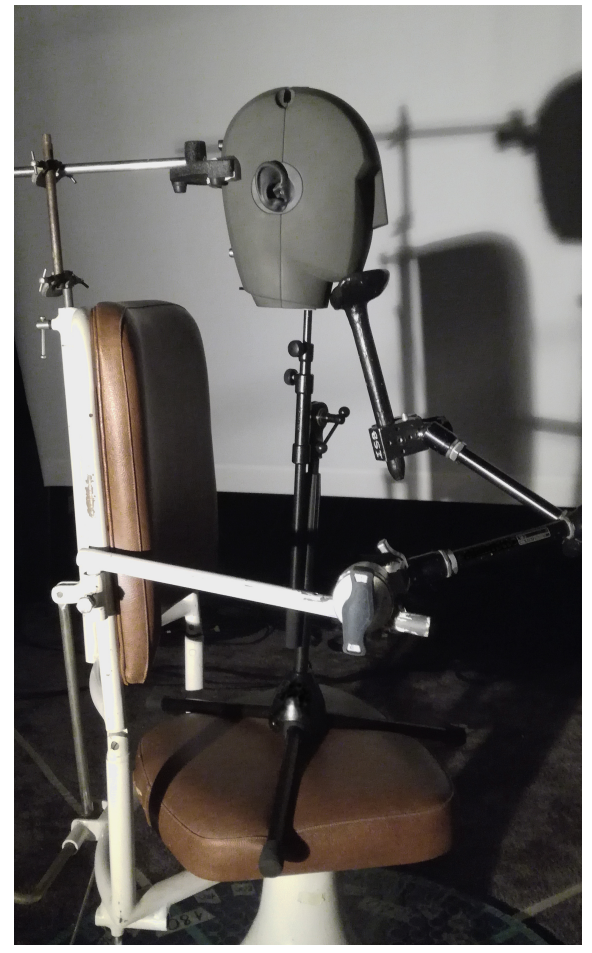

Fig. 3. Position of the dummy head, maintained by the headrest and chin rest, during the recording of the stimuli.

jects were asked to place the test headphones comfortably over their ears and to not modify this position once the test had started as realistic changes in the headphone position can be audible [27]. Subjects had to carry out eight loudness equalizations, corresponding to the two equalizations (one for each test sound) in each of the four spatial configurations of the session 1 (see Fig 2).

In order to compare the effect of the sole ITD on the loudness of pure tones to that of the narrow-band noise used as stimulus in this experiment, one additional equalization was realized in this session. Its reference sound was the narrow-band noise directly synthesized and played back through the headphones (without being recorded by the dummy head) in a diotic presentation, at the same reference level than the previous stimuli (50 phon). The test sound that was to be equalized on this reference sound was the same narrow-band noise directly synthesized with a fixed $772 \mu$ s time difference applied between the two channels. This value was also used in the previous experiments on the effect of ITD on loudness [9, 11, 12].

These nine equalizations were done in the same session that lasted approximately $30 \mathrm{~min}$. Stimuli appeared in a random order for each subject.

\section{Results}

Since the PSE is the level difference for which a test sound is perceived as loud as a reference sound, a negative PSE means that a lower sound pressure level was required for the test sound to match the reference sound in loudness. Thus, when providing both sounds with the same pressure level, the test sound would have been perceived louder than 
the reference sound, indicating a higher loudness sensitivity to directional sounds. The results are subsequently presented as DLS for the rest of the paper:

$$
\mathrm{DLS}=-\mathrm{PSE}
$$

The outcome of the ninth equalization (involving diotic and dichotic headphone-synthesized stimuli) presented in the same session as the virtual presentation does not properly result from the comparison of real sources located in different directions but from sounds differing in their ITDs only. This loudness match will also be denoted as DLS for sake of clarity.

In order to investigate the effect of the presentation conditions on directional loudness, a repeated-measures analysis of variance (ANOVA) was conducted on the sixteen DLS values obtained in actual and virtual presentations. The spatial configuration (4 levels) and the test sound azimuth (2 levels) were also included as factors of the analysis:

- Presentation conditions (2 levels): actual, virtual.

- Spatial configuration (4 levels): configurations $a, b, c, d$.

- Azimuth (2 levels): $-90^{\circ},+90^{\circ}$.

Results of this ANOVA are given in Table 1 . As this study primarily focuses on the effects of the presentation conditions on the DLS, the significant effects involving this factor will be discussed first. Other significant effects, namely the "spatial configuration" effect and its interaction with the sound source azimuth are caused by the room effect. The main consequences of this room effect is that the DLS values themselves are not consistent with the loudness gain provided by the lateralization of a sound source in one given spatial configuration within the room, leading to an inconsistent pattern across the configurations identifiable on Fig. 4 and Fig. 5 . However, the room effect is likely to be cancelled when averaging DLS values across the spatial configurations, and it did not have any influence on the presentation conditions effects since it was globally the same whether the sounds were reproduced over headphones or loudspeakers, and was therefore present in a similar way in the actual and virtual presentation conditions.

\subsection{Presentation conditions effects}

One of the main results regarding this analysis is that the presentation conditions proved to have significant effects on the DLS. These effects were dependent on the spatial configuration and on the test sound azimuth, as indicated by the significant "presentation conditions $\times$ spatial configuration $\times$ azimuth" interaction. Bonferroni posthoc tests revealed a significant difference between the two presentation conditions in the spatial configuration $c$ when the test sound was located at $-90^{\circ}(p<.001)$ or at $+90^{\circ}$ $(p=.007)$. In this configuration and for these two azimuths, DLS was larger in the virtual presentation than in the actual presentation, as shown in Fig. 4.

Presentation conditions effects also appeared to depend on the spatial configurations when combining the test sound azimuths as the "presentation conditions $\times$ spatial configuration" interaction proved to be significant. Thus, the effect of the presentation conditions on DLS was not the same for all spatial configurations of the sound sources. Bonferroni post hoc tests revealed a significant difference between the two presentation conditions in the spatial configurations $c(p<.001)$ and $d(p=.016)$ only. In these two configurations, DLS was larger in the virtual presentation than in the actual presentation, as shown in Fig. 5. As depicted in Fig. 2, the configurations $c$ and $d$ were similar in the way that the reference sound source was at a distance of $1 \mathrm{~m}$ from the subject, whereas it was at a distance of $2 \mathrm{~m}$ in configurations $a$ and $b$. Then, the fact that DLS did not significantly depend on the presentation conditions in the configurations $a$ and $b$ whereas they did significantly depend on the presentation conditions in the configurations $c$ and $d$ is likely to be caused by the distance of the frontal sound source.

To summarize about these two interactions :

- DLS was similar whether the sound sources were actual or virtual when the frontal sound source was located at $2 \mathrm{~m}$ from the subjects.

- Virtual sound sources yielded to larger DLS than actual ones when the frontal sound source was located at $1 \mathrm{~m}$ from the subjects, for each of the two azimuths in the configuration $c$ and when combining the two azimuths in the configuration $d$.

Finally, the presentation conditions proved to have a significant effect when combining all spatial configurations and azimuths, as shown by the significance of the "presentation conditions" simple effect. As indicated in the left part of Fig. 6, DLS was significantly larger with the virtual presentation of the sounds than with the actual one. The right part of the figure depicts the DLS obtained for the noise synthesized and directly reproduced over headphones with a fixed $772 \mu$ s ITD between the two channels. A $t$-test revealed that this DLS is significantly larger than zero $(t(19)=5.296, p<.001)$ and is about $1.5 \mathrm{~dB}$, which is in agreement with the previous findings on the effect of ITD on loudness for pure tones [9, 11, 12]. In addition, independent-samples $t$-tests revealed that neither the DLS obtained in virtual $(t(29.949)=1.501], p=.144)$ nor actual $(t(178)=-.531, p=.596)$ presentation conditions is significantly different from the DLS of the stimulus with a fixed ITD. The DLS was also significantly larger than zero in both virtual $(t(159)=13.677, p<.001)$ and actual $(t(159)=11.433, p<.001)$ presentation conditions.

At the frequencies under investigation, the fact that the DLS is larger than zero is likely to be accounted for by the ITD. Then, the average time difference between the two channels of the dummy head was measured for each test sound (two azimuths and four spatial configurations) in order to be compared to the fixed $772 \mu$ s ITD. These eight

\footnotetext{
${ }^{1}$ corrected due to a violated assumption of homogeneity of variances.
} 


\begin{tabular}{|l||c|c|c|c|c|}
\hline Source & SS & DF & MS & F & Sig.p \\
\hline \hline Presentation conditions & 35.099 & 1 & 35.099 & 19.946 & $<.001$ \\
\hline Spatial configuration & 182.546 & 3 & 60.849 & 59.740 & $<.001$ \\
\hline Azimuth & .023 & 1 & .023 & .017 & .899 \\
\hline Presentation conditions $\times$ spatial configuration & 29.282 & 3 & 9.761 & 11.671 & $<.001$ \\
\hline Presentation conditions $\times$ azimuth & .183 & 1 & .183 & .234 & .634 \\
\hline Spatial configuration $\times$ azimuth & 265.726 & 3 & 88.575 & 76.301 & $<.001$ \\
\hline Presentation conditions $\times$ spatial configuration $\times$ azimuth & 10.358 & 3 & 3.453 & 4.170 & $\boldsymbol{p}=. \mathbf{0 1}$ \\
\hline
\end{tabular}

Table 1. ANOVA results.

measurements led to a slightly higher average ITD than $772 \mu \mathrm{s}(\mu=859 \mu \mathrm{s}, \sigma=19 \mu \mathrm{s})$, but should not lead to a significantly different DLS [28].

\subsection{Spatial configuration effects}

The ANOVA also revealed that the spatial configuration had significant effects on the DLS values. In other words, the loudness ratio between lateral and frontal sound sources was not the same depending on the spatial configuration of the sound sources in the listening room. Since this room was not anechoic, these effects are likely to be caused by sound pressure variations at the ears of the listeners and of the dummy head even though the four loudspeakers were calibrated to deliver the same level at the center of the subjects heads and of the dummy head. Therefore, atear SPL variations affecting frontal and lateral sources will also affect the DLS values resulting from the comparison of these two sources, as indicated by the "spatial configuration" simple effect and by the "spatial configuration $\times$ azimuth" interaction. As an example, a spatial configuration for which the reference sound pressure level is lower than that of the reference sound in another spatial configuration would lead to higher overall DLS values. In order to verify this hypothesis, the reference sound pressure levels were measured on each ear of the dummy head in each spatial configuration and averaged over the two ears, before being compared to the overall DLS value in each spatial configuration. The results are depicted on Fig. 7, and show a good match between the two patterns. These SPL variations (Fig. 7 right) appear to partially account for the DLS dependency on spatial configuration (Fig. 7l left), although one should keep in mind that the SPL of the lateral test sound is also involved in DLS. It should be emphasized that the Y-axis of the right figure is reversed since reference sound pressure level and DLS values are anticorrelated. The $0 \mathrm{~dB}$ in this figure is the level delivered by the reference sources (50 phon) at the center of the head.

The DLS value in each spatial configuration may then per se not be representative of the directional loudness effect in a given configuration because of these SPL variations. On one hand, if a loudspeaker delivered a lower SPL than its neighbour at the ears of the listeners and of the dummy head, this results in a higher DLS value in the spatial configuration in which it is the reference source. On the other hand, the DLS value in the spatial configuration where its neighbour is the reference (see Fig. 1 and Fig. 2) will be proportionally lower. Thus, the room effect is likely to be averaged over the four spatial configurations. This is supported by the good match between the averaged DLS obtained with the room acoustic and the DLS obtained with ITD only (Fig. 6) since at the frequencies under investigation, directional loudness is expected to be mostly accounted for by ITD.

In addition, these at-ear SPL differences were present in the two presentation conditions because of the binaural recording method employed for creating virtual sound sources. Inter-individual SPL variations may have resulted from the coupling between the headphone and each individual listener's ears, but the average at-ear SPLs were assumed to follow a similar pattern as the one measured by the dummy head and depicted on the right of Fig. 7 In conclusion, these SPL differences are not likely to account for the observed effects of the presentation conditions on the DLS.

\section{Discussion}

The results of the experiment show that:

- The loudness of a low-frequency narrow-band noise was higher when it was located at the side of a listener than when it was located at the front, for the two presentation conditions under investigation.

- This increase was not different, for both presentation conditions, from the increase observed with ITD only.

- The increase was inferior in the actual presentation (sounds stemming from actual loudspeakers) than in the virtual presentation (binaural recording of the loudspeaker-reproduced stimuli, with no loudspeakers mounted in the tested directions) when the frontal sound source was at a distance of $1 \mathrm{~m}$ from the subject.

- The increase was the same in both presentation conditions when the frontal sound source was at a distance of $2 \mathrm{~m}$ from the subject.

- For the positions were the frontal sound source was at a distance of $1 \mathrm{~m}$, this increase occurred for each of the two lateral source azimuths in configuration $c$ whereas it occurred only when combining the two azimuths in configuration $d$.

\subsection{Effect of the presentation conditions}

The overall DLS obtained in the actual presentation conditions was lower than the DLS obtained in the virtual presentation conditions. This result is in agreement with those 

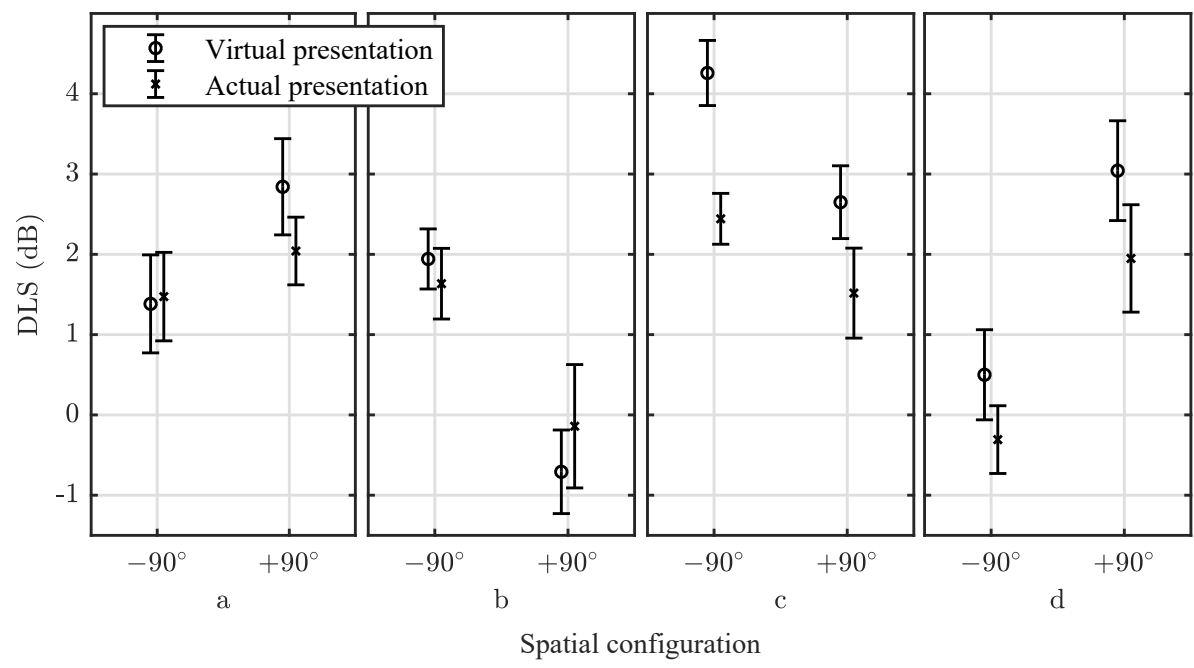

Fig. 4. DLS as a function of the azimuth of the test sound in each spatial configuration for the two presentation conditions, with $95 \%$ confidence intervals.

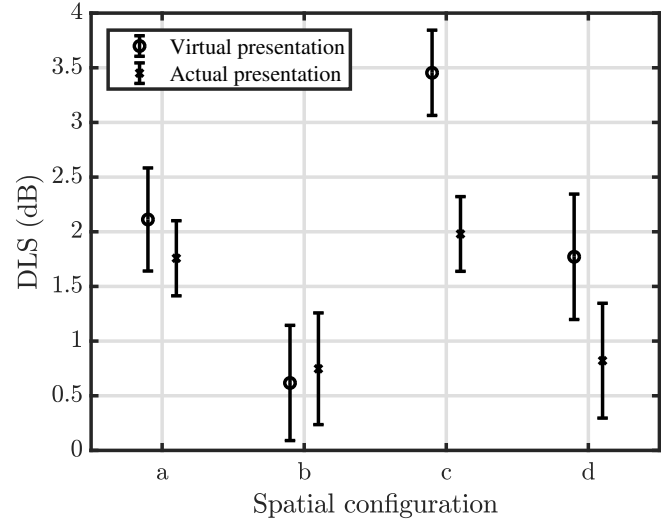

Fig. 5. DLS as a function of the spatial configuration for the two presentation conditions, with $95 \%$ confidence intervals.

obtained by Epstein and Florentine [13, 14], who measured a lower binaural-to-monaural ratio when increasing the ecological validity of their experiments by displaying stimuli through loudspeakers rather than through headphones and by adding visual cues to their stimuli. As a result, binaural or directional loudness investigations achieved under controlled laboratory presentation conditions are likely to highlight isolated phenomenons that affect binaural loudness summation but whose observed magnitude could be higher than when observed in real life. Comparatively, experiments designed using real sound sources along with visual cues are likely to highlight closer to natural magnitude effects.

According to the results of the present study, the distance of the frontal sound source (displaying the reference sound) had a different effect on DLS in the two presentation conditions. Since both auditory [29] and visual [30] distance cues were reported to affect loudness, these distance cues could account for the variation of DLS with distance.

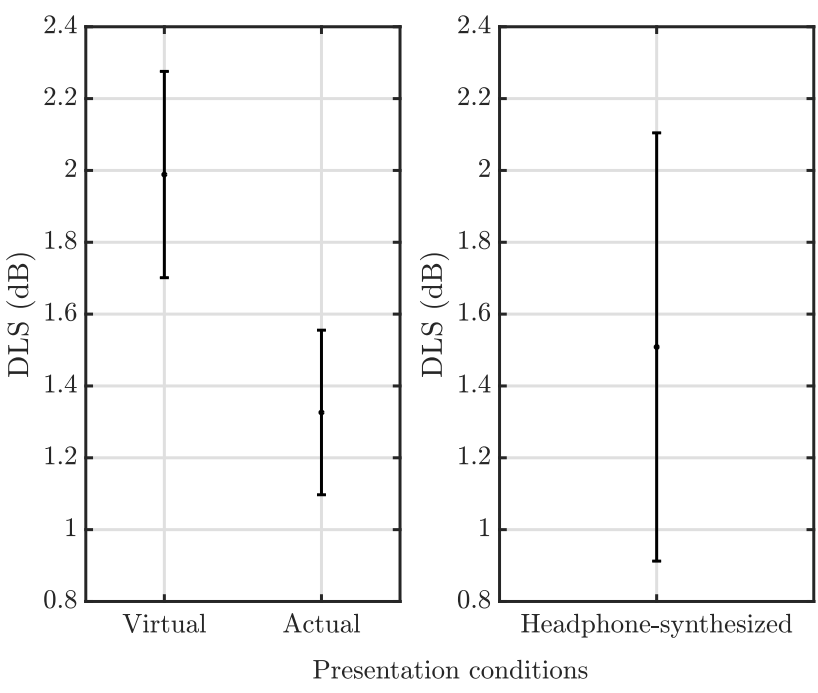

Fig. 6. DLS as a function of the presentation conditions (left) and for headphone-synthesized stimuli (right), with $95 \%$ confidence intervals.

\subsection{Auditory cues}

Since the SPL was fixed at a same listening point for the four loudspeakers, it did not vary whether the sound sources were at 1 or $2 \mathrm{~m}$ from the subjects and could not account for DLS variations. On the other hand, other acoustic characteristics of the sound could be affected by the distance of the sources (i.e., direct-to-reverberant ratio, spectral energy distribution...) [7]. Those characteristics were similar in the two presentation conditions thanks to the binaural recording used in the virtual presentation. Some differences might however have resulted from the differences between the subjects' individual HRTFs (that filtered the sounds in the actual presentation) and the dummy head HRTFs (that filtered the sounds in the virtual presentation). Yet, HRTFs depend on source distance only in proximal space $(<1 \mathrm{~m}$ and even closer at the frequencies under investigation) [31, 32]. Then, even if a different filtering oc- 


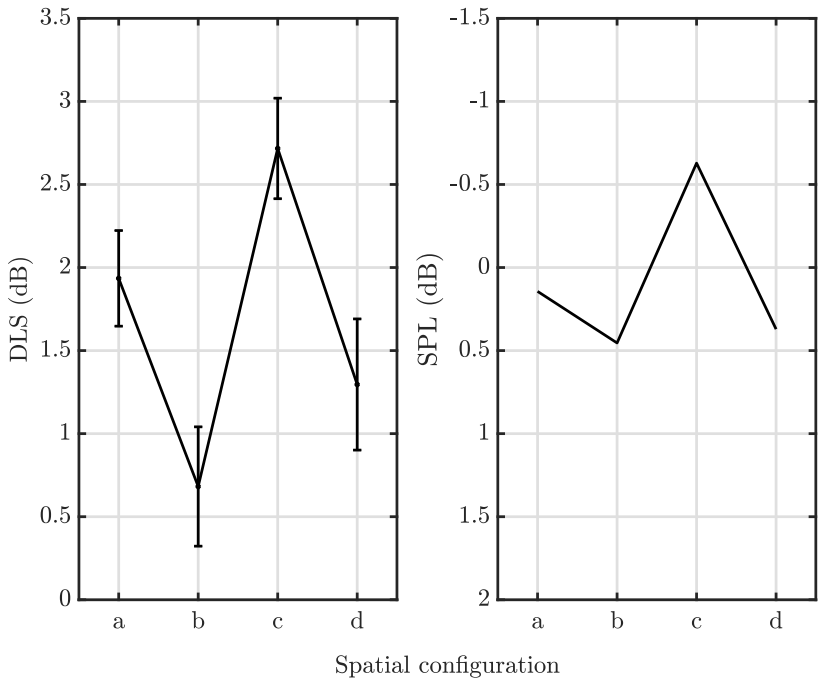

Fig. 7. DLS as a function of the spatial configuration (left) compared to the average SPL between the two ears of the dummy head for the reference sound (right) in each spatial configuration.

curred in the actual and virtual presentation conditions, the differences should remain the same whether the source is at 1 or $2 \mathrm{~m}$ from the subjects.

Furthermore, it could be considered that the DLS dependency on source distance was not determined by the auditory distance cues themselves but by the resulting perceived distance. Then, a potential effect of visual cues to distance should also be discussed since visual estimates of distance are better than auditory estimates [33].

\subsection{Visual cues}

The visual cues to distance were not the same in the two presentation conditions. In the virtual presentation, neither frontal nor lateral sound sources were visible and subjects had little if any clue to physical sources that may have reproduced the sounds they heard. In the actual presentation, visual cues for the frontal sound sources were available to the subjects during the whole procedure, which was not the case for the lateral sound sources because of the headrest and chin rest that maintained their heads in a fixed position. Thus, subjects did not have the same access to visual information according to the presentation conditions and to the azimuth of the sources.

When the frontal sources were visible and close to the subject (that is in the actual presentation conditions in the spatial arrangements $c$ and $d$, see Fig. 2), DLS was significantly lower than when they were not visible (that is in the virtual presentation conditions). This decrease means that the loudness of the reference and test sounds tended to get closer. Since DLS was positive (i.e., test sounds were perceived louder than reference sounds), the DLS lowering could be due either to an increase in loudness of the reference sound or to a decrease in loudness of the test sound. In each spatial configuration of the sources, DLS was assessed on both 1 and $2 \mathrm{~m}$ distant lateral test sounds. Thereby, if the distance of the lateral sound source affected DLS in any way, its effect would be canceled in each spatial configuration because of the assumed left/right symmetry of directional loudness [9]. Thus, the observed DLS differences between the two presentation conditions are more likely to be accounted for by the loudness of the frontal reference sounds. Following this hypothesis, the loudness of sounds that come from visible and close sources would be higher than the loudness of sounds that come from invisible or farther sources at a same physical level delivered to the listener. Such an effect was reported for visible sources in an anechoic room: the loudness related to a loudspeaker located at $3.75 \mathrm{~m}$ was less than when at $2.25 \mathrm{~m}$, even though the sound pressure level was fixed at the position of the listener [30]. This explanation could also account for the fact that no DLS difference was observed between the loudspeaker and headphone presentation conditions investigated by Sivonen et al. [2, 16] since visual cues were provided in the two conditions.

\subsection{Other factors}

In the present experiment, listeners were aware that the sounds they heard were displayed whether by loudspeakers or by headphones in the corresponding test sessions. This awareness could have had an effect on the loudness processing of the sounds [14], independently from physical changes in the heard stimuli, and the exact same sounds could somehow have led to different DLS judgments according to the presentation conditions. Distance perception could also have interacted with other high level factors, for example the externalization of the sound sources. Even though relations between externalization of a sound source (i.e., perceiving a sound as coming from the exterior of the head) and perceived distance are not fully established yet [34], it could be hypothesized that sound externalization played a role in the observed loudness summation. Sounds are likely to be internalized (i.e. perceived as coming from "inside" the head) when they are reproduced over headphones, especially when displayed at the front $\left(0^{\circ}\right)$ than at the side $\left( \pm 90^{\circ}\right)$ of a listener [35, 36]. On the other hand, sounds reproduced over actual sound sources are reported to be externalized whatever their location with respect to the listener [37]. Then, the degree of externalization could not have been the same for reference (located at $0^{\circ}$ ) and test sound (located at $\pm 90^{\circ}$ ) sources in the virtual presentation conditions, while it should have been the same for all sound sources in the actual presentation conditions. This could have had an effect on the perceived sound sources distance, and as a consequence on the resulting DLS.

\section{Conclusion}

The results of this experiment extended the previously highlighted effect of ITD on loudness of stimuli displayed under unrealistic listening conditions to that of stimuli reproduced with natural binaural cues in a listening context with stronger ecological validity.

The directional loudness effect was found to be dependent on the presentation conditions in which the sounds were perceived. More precisely, the loudness of sounds was 
increased by a higher amount when they were side-located and heard in virtual presentation conditions with no visible sound source than when they were presented by actual sound sources located at the side of the listener. This only occurred when the frontal sound source, used as a reference, was located at the closest studied distance $(1 \mathrm{~m})$ from the subject. One hypothesis accounting for these observations is that for a same sound pressure level, sounds produced by frontal sources could have been perceived louder when the sources were located at $1 \mathrm{~m}$ from the subject than when they were located at $2 \mathrm{~m}$ from the latter, provided the sound sources were visible. This condition was only fulfilled in the actual presentation conditions. These DLS differences could also be accounted for by other parameters that varied between the two presentation conditions, such as the HRTFs used, or more globally by high level processes that rely on the presentation conditions themselves rather than on their effects on the stimuli.

\section{Acknowledgments}

The authors wish to thank the staff from the "Image \& Son" department of the University of Brest and all the participants of this experiment.

This work was supported by the ANR-13-CORD0008 EDISON 3D project, funded by the French National Agency of Research (http://www. agence-nationale-recherche.fr/).

\section{REFERENCES}

[1] D. W. Robinson, L. S. Whittle, "The loudness of directional sound fields," Acta Acust. united Ac., vol. 10, no. 2, pp. 74-80 (1960).

[2] V. P. Sivonen, W. Ellermeier, "Directional loudness in an anechoic sound field, head-related transfer functions, and binaural summation," J. Acoust. Soc. Am., vol. 119, no. 5, pp. 2965-2980 (2006), doi:https://doi.org/10.1121/ 1.2184268

[3] V. P. Sivonen, "Directional loudness and binaural summation for wideband and reverberant sounds," $J$. Acoust. Soc. Am., vol. 121, no. 5, pp. 2852-2861 (2007), doi:https://doi.org/10.1121/1.2717497.

[4] V. P. Sivonen, W. Ellermeier, "Binaural loudness for artificial-head measurements in directional sound fields," $J$. Audio Eng. Soc., vol. 56, no. 6, pp. 452-461 (2008).

[5] N. Zacharov, O. Tuomi, G. Lorho, "Auditory periphery, HRTF's and directional loudness perception," presented at the 110th Convention of the Audio Engineering Society Convention (2001 May), convention paper 5315.

[6] I. Dash, L. Miranda, D. Cabrera, "Multichannel Loudness Listening Test," presented at the 124th Convention of the Audio Engineering Society Convention (2008 May), convention paper 7451.

[7] B. C. J. Moore, "Space perception," in An Introduction to the Psychology of Hearing, pp. 245-250 (BRILL, Leiden, Netherlands) (2012).

[8] B. C. J. Moore, B. R. Glasberg, A. Varathanathan, J. Schlittenlacher, "A loudness model for time-varying sounds incorporating binaural inhibition," Trends Hear., vol. 20 (2016), doi:https://doi.org/10.1177/ 2331216516682698.

[9] V. Koehl, M. Paquier, "Loudness of low-frequency pure tones lateralized by interaural time differences," $J$. Acoust. Soc. Am., vol. 137, no. 2, pp. 1040-1043 (2015), doi:https://doi.org/10.1121/1.4906262.

[10] G. F. Kuhn, "Model for the interaural time differences in the azimuthal plane," J. Acoust. Soc. Am., vol. 62, no. 1, pp. 157-167 (1977), doi:https://doi.org/10.1121/1. 381498.

[11] V. Koehl, M. Paquier, E. Hendrickx, "Effects of interaural differences on the loudness of low-frequency pure tones," Acta Acust. united Ac., vol. 101, no. 6, pp. 11681173 (2015), doi:https://doi.org/10.3813/AAA.918909.

[12] G. Berthomieu, V. Koehl, M. Paquier, "Influence of interaural time differences on loudness for low-frequency pure tones at varying signal and noise levels," Proc. Mtgs. Acoust., vol. 30, p. 050004 (2017), doi:https://doi.org/10. 1121/2.0000553.

[13] M. Epstein, M. Florentine, "Binaural loudness summation for speech and tones presented via earphones and loudspeakers," Ear Hear, vol. 30, no. 2, pp. 234-237 (2009 Apr.), doi:https://doi.org/10.1097/AUD. 0b013e3181976993.

[14] M. Epstein, M. Florentine, "Binaural loudness summation for speech presented via earphones and loudspeaker with and without visual cues," J. Acoust. Soc. Am., vol. 131, no. 5, pp. 3981-3988 (2012 May), doi: https://doi.org/10.1121/1.3701984.

[15] J. Blauert, "Distance hearing and inside-the-head locatedness," in Spatial Hearing: The Psychophysics of Human Sound Localization, p. 116 (MIT Press, Cambridge, MA) (1997).

[16] V. P. Sivonen, P. Minnaar, W. Ellermeier, "Effect of direction on loudness in individual binaural synthesis," presented at the 118th Convention of the Audio Engineering Society (2005 May), convention paper 6512.

[17] E. H. A. Langendijk, A. W. Bronkhorst, "Fidelity of three-dimensional-sound reproduction using a virtual auditory display," J. Acoust. Soc. Am., vol. 107, no. 1, pp. 528537 (2000), doi:https://doi.org/10.1121/1.428321.

[18] B. R. Glasberg, B. C. J. Moore, "Derivation of auditory filter shapes from notched-noise data," Hear. Res., vol. 47, no. 12, pp. 103-138 (1990), doi:https://doi.org/10. 1016/0378-5955(90)90170-T.

[19] B. A. Edmonds, J. F. Culling, "Interaural correlation and the binaural summation of loudness," J. Acoust. Soc. Am., vol. 125, no. 6, pp. 3865-3870 (2009), doi: https://doi.org/10.1121/1.3120412.

[20] ISO 226, "Acoustics - Normal equal-loudnesslevel contours," (2003).

[21] ANSI S3.21, "Methods for manual pure-tone threshold audiometry," (2004).

[22] H. Levitt, "Transformed updown methods in psychoacoustics," J. Acoust. Soc. Am., vol. 49, no. 2B, pp. 467-477 (1971), doi:https://doi.org/10.1121/1.1912375.

[23] ISO 3382-2, "Measurement of room acoustic parameters - Reverberation time in ordinary rooms," (2008). 
vol. 65, pp. 589-599 (2017b), doi:https://doi.org/10.17743/ jaes.2017.0018.

[37] W. M. Hartmann, A. Wittenberg, "On the externalization of sound images," J. Acoust. Soc. Am., vol. 99, no. 6, pp. 3678-3688 (1996), doi:https://doi.org/10.1121/ 1.414965 .

\section{THE AUTHORS}

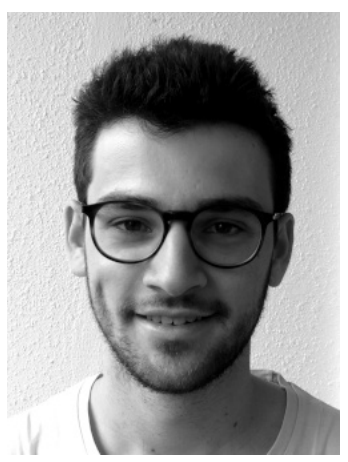

Gauthier Berthomieu

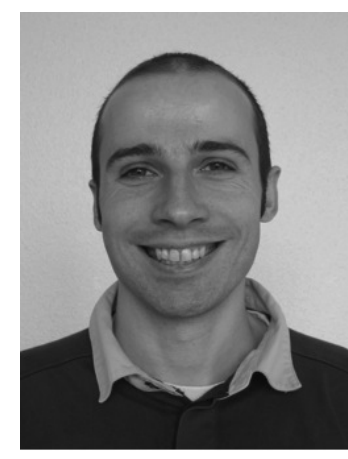

Vincent Koehl

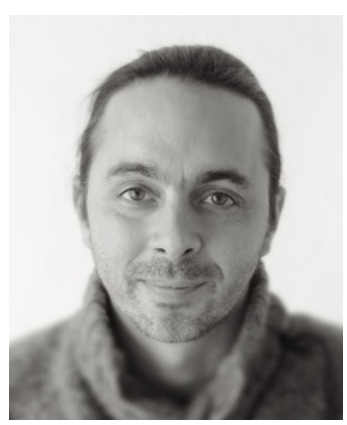

Mathieu Paquier
Gauthier Berthomieu was born in Mont-de-Marsan, France, in 1994. He received the M.Sc. degree in sound engineering from the "Image \& Sound" department of the University of Brest in 2016. Since 2016, he is a Ph.D. student at the University of Brest where he works on 3D sound perception and more particularly on directional loudness. $\mathrm{He}$ is a member of the Audio Engineering Society.

Vincent Koehl was born in Créhange, France, in 1978. He graduated from the National Institute for Applied Sciences (INSA) of Lyon (France) in Mechanical Engineering in 2001 (M.Sc. degree) and received a Masters Degree in Acoustics in 2002. In 2005, he received a Ph.D. degree for his work about the effects of structural uncertainty on sound quality accomplished at the Laboratory for Vibration and Acoustics (LVA). Vincent Koehl is now Associate Pro- fessor at the University of Brest, France. His main research interests are the perceived quality of sound reproduction systems, 3D sound perception, spatial audio recording and rendering technique. Dr. Koehl is a member of the French Acoustical Society, elected to the Board of the Sound Perception Group.

Mathieu Paquier was born in Bordeaux, France, in 1974. He received the M.Sc. degree in sound engineering from the University of Brest in 1997, then a Ph.D. degree in acoustics from the University of Lyon in 2002. He is Professor and head of the "Image \& Sound" department of the University of Brest since 2016. Pr. Paquier's research interests mainly include the evaluation of spatial sound and the perception of headphone restitution. 